\title{
Editorial \\ Special Issue on Novel Approaches for Structural Health Monitoring
}

\section{Cecilia Surace}

Citation: Surace, C. Special Issue on Novel Approaches for Structural Health Monitoring. Appl. Sci. 2021, 11, 7210. https://doi.org/10.3390/ app11167210

Received: 27 July 2021

Accepted: 1 August 2021

Published: 5 August 2021

Publisher's Note: MDPI stays neutral with regard to jurisdictional claims in published maps and institutional affiliations.

Copyright: (C) 2021 by the author. Licensee MDPI, Basel, Switzerland. This article is an open access article distributed under the terms and conditions of the Creative Commons Attribution (CC BY) license (https:// creativecommons.org/licenses/by/ $4.0 /)$.
Department of Structural, Geotechnical and Building Engineering, Politecnico di Torino, 10129 Turin, Italy; cecilia.surace@polito.it

\section{Introduction}

Crucial mechanical systems and civil structures or infrastructures, such as bridges, railways, buildings, wind turbines, aeroplanes and more are subjected during their lifetime to natural deterioration of their structural integrity. This is due to several concomitant factors (i.e., environmental conditions, operating loads, etc.). Worsening of this degradation or accidental events lead to an impairment of the functionality and a severe decrease of the safety level, while extremely critical interruptions of service and catastrophic collapses with possible loss of life may occur. Moreover, taking into consideration also the economic relevance of these systems, sustainable management requires the implementation of specific maintenance strategies to reduce the related repair costs.

Due to all these reasons, the last decades have seen a growing interest in the field of structural health monitoring (SHM), involving multidisciplinary academics and practitioners aimed to develop effective technologies, procedures and algorithms for damage diagnosis. Continuous scientific advancement and technological evolution provide the means to face old and new challenges. These are overcome by designing new approaches, defining new damage-sensitive features, and enhancing the already-existing devices for this scope. However, although in recent years numerous interesting findings and applications have been made, further efforts are still needed.

In this context, the "Special Issue on Novel Approaches for Structural Health Monitoring" in Applied Sciences collects 15 published papers, each of them a relevant contribution on a specific related fundamental aspect. These are briefly reviewed here for the reader's convenience.

\section{The Extreme Function Theory for Damage Detection: An Application to Civil and Aerospace Structures}

The research reported in [1] describes an approach, based on the extreme function theory (EFT), for mode shape-based damage detection. Applications to both aerospace and civil structures are proposed as (numerical and experimental) case studies. The EFT can be considered as the extension of the classic extreme value theory (EVT) to whole functions-in this specific case, to mode shapes, extracted from the target system and benchmarked against the ones identified from the pristine baseline. More into detail, the continuous mode shapes are defined by means of Gaussian process regression (GPR), applied over a limited set of data points. These correspond to the output channels distributed over the structure under investigation. To compensate for the shortage of training data, a data augmentation strategy is included as well. This is intended to deal with the practical issue of data scarcity, which often hampers the applicability of machine learning approaches for SHM. The performances of the procedure are addressed in terms of true positives, true negatives, and type I and II errors. The rationale is to investigate not only the damage detection capabilities of the algorithm but also its robustness to false alarms. The robustness to artificially added measurement noise is tested as well on several finite element models - a simple beam with several boundary conditions, the spar of a prototype 
high aspect ratio wing, and a shear-type 3-stories frame structure. Finally, a direct comparison between EFT-based damage detection and the EVT-based alternative is reported, considering in the latter case the same algorithm applied pointwise only at the output channels. This last study shows a statistically significant reduction of false alarms.

\section{Full-Field Strain Reconstruction Using Uniaxial Strain Measurements: Application to Damage Detection}

The accurate full-field reconstruction of the strain and displacement fields of a structure using a set of uniaxial strain measurements is the prime focus of the work presented in [2]. The use of uniaxial strain measurements, as obtained from a fibre-optic sensor, for two-dimensional displacement field reconstruction leads to difficulties due to insufficient strain information at a point and can potentially lead to a breakdown of the reconstruction procedure. This work proposes a solution to the problem based on the inverse finite element method (iFEM), combined with a pre-processing step for strain smoothing using the smoothing element analysis (SEA) approach. The iFEM is a variationally-based approach, where the structural domain is discretized using finite elements, and the displacement field is reconstructed by minimizing an error functional representing the least-squares error between analytic and experimental strain measures. The effect of sensor position and orientation on reconstruction results is investigated and used to identify effective strain-sensor patterns ensuring reconstruction accuracy. The iFEM performance is evaluated numerically using the problem of a thin plate, subject to several internal damage scenarios. Damage detection capability depends on an accurate reconstruction of the local internal strain perturbations, and the iFEM reconstructed strain fields successfully revealed the damage locations as regions of strain concentration containing information regarding damage size, position, and orientation. Additionally, a sensitivity analysis demonstrates the proposed methodology's robustness to measurement noise, although it hinted at difficulties in detecting small-sized damages. The main achievement of the paper is in showing the potential of strain measurements based on fibre optic sensors for practical SHM applications.

\section{Rail Diagnostics Based on Ultrasonic Guided Waves: An Overview}

The diagnostic of rail tracks damage conditions is the topic investigated in [3]. The authors give an extensive overview from the general context to the latest innovations, focusing on different non-destructive testing (NDT) methodologies. The authors describe the implementation and analysis of the performance of inspection strategies based on ultrasonic guided waves (UGW). Firstly, a detailed introduction about various types of rail track defects and different rail diagnostic techniques developed in the last decades is reported. Then, with a proper distinction between the main classes of diagnostic systems (on-board, land-based) and types of approaches (active, passive), the specific architecture and data processing approaches related to UGW methodologies are presented. Next, comprehensive sections are explicitly dedicated to the latest land-based systems, dealing with core systems of ultrasonic broken rail detector, early rail defect detection capability, mixed evolved techniques and other commercial projects. Finally, the performance analyses of all the aforementioned rail diagnostics are shown, followed by a discussion about their advantages and disadvantages. The authors additionally outline several potential future developments based on the limits or open issues of current, state-of-the-art ultrasonic systems. For its completeness and detailed considerations, the works presented represents an effective baseline for new researches and further improvements.

\section{Mooring-Failure Monitoring of Submerged Floating Tunnel Using Deep Neural Network}

A study of structural health monitoring of submerged floating tunnels (SFT) is presented in [4]. This kind of infrastructure presents many advantages in comparison with suspended or floating bridges since these latter solutions are very responsive to environmental solicitations (wave, seismic actions, etc...). Moreover, SFTs naturally allow sea crossing without any physical impediment. Nevertheless, due to their submerged condi- 
tion, any failure occurrence of the balancing mooring can be catastrophic if not promptly repaired. Therefore, the monitoring and maintenance of these infrastructures result challenging and expensive. Thus, the authors propose a deep learning-based algorithm, able to overcome all the limitations of traditional maintenance strategies such as the need for visual inspection, the requirement of numerous sensors, or the prior knowledge of structural parameters. In particular, a deep neural network (DNN) has been implemented to analyse lateral and vertical displacements measured through different accelerometers settings under different wave conditions. Numerical simulations have been initially used to select the best features of the neural network, as its architecture (i.e., the number of hidden layers and neurons), the activation function, and the loss function. The different investigations show that a very high rate of accuracy for correct detection can be reached with a reasonably low number of sensors (from three to seven if opportunely located). These results have been confirmed under different wave conditions and by comparing them with experimental results. The work presented here, thus, enhances the feasibility perspective in SFT spread, since it deploys an effective and attainable method for a valid monitoring strategy.

\section{Damping of Beam Vibrations Using Tuned Particles Impact Damper}

The work presented in [5] introduces an innovative tuned particle impact damper (TPID). The device is proposed as an improvement of the well-known particle impact damper vibration adsorbers, with the main feature of rapid tuning its damping parameters. The dissipation of the kinetic energy occurs through friction and viscous and inelastic impacts of the particles among themselves and the container. The governing mechanism of damping depends on several factors, such as geometry, material, mass and stiffness of the grains, coefficient of restitution, the composition of the container, filling ratio, particles degradation, and temperature increase. These are investigated by experimental verifications. The conclusions show that the most relevant parameters are the volume ratio between the grains and the container. The authors propose a simple yet highly efficient design: the grains are encapsulated into an inflatable balloon provided by an external valve, allowing the easy and quick tuning of the available volume by varying the internal pressure. Numerous tests and experiments assess the characteristics of the novel device. Results show that damping performances increase when dealing with a higher mass of grains and higher balloon volume. Regarding the composition of the grains, a plastic material is found as the best solution among the options investigated by the authors. Indeed, the selected material proved to be very responsive to volume variations while ensuring high vibration attenuation. In conclusion, this study lays the basis for a truly innovative, low-cost and ecologically sustainable device, enhancing the opportunities of semi-active vibration attenuation strategy thanks to its tunable damping feature.

\section{Health and Structural Integrity of Monitoring Systems: The Case Study of Pressurized Pipelines}

The authors of [6] present the physical principles of structural health monitoring related to the specific topic of pressurised pipelines, giving a strong overview supported by multiannual experience in the field and real results coming from operating systems. The main issues causing damages in pressurised pipelines (which often result in catastrophic failures) are traced back to unpredictable variations in operating conditions, such as changes in internal pressures, landslides, subsidence of foundation ground soil, or steel corrosion. Moreover, these root causes involve secondary effects like induced vibrations and additional stresses, often interacting simultaneously. Since the unpredictability of these phenomena implies the impossibility to provide adequate design solutions, the authors individuate in long-term real-time monitoring systems the only feasible tool to overcome these issues. In particular, stress and strain measurements are envisaged as fundamental to determine high-risk conditions while taking into consideration some crucial guidance. For instance, fatigue cycles should be analysed individually in order to properly assess the anelasticity limit for fatigue crack. Sensing devices applied for long-term monitoring must 
appropriately compensate for the thermal effects to be reliable. In the case of additional stress generated by soil movements, the sensors location setting should cover the whole circumference for a complete observation of the stress field. Finally, corrosion insurgence has to be rightly avoided with opportune protection and continuously monitored, since it can cause wall weakening and sudden collapses. The possible choices for its monitoring include analytical solutions of kinematic growth equations or prescriptions from current normative. Ultimately, the guidelines provided by the authors in this paper give useful considerations resulting from direct field experience.

\section{Strain Response Characteristics of RC Beams Strengthened with CFRP Sheet Using BOTDR}

The research reported in [7] focuses on the crucial aspect of monitoring reinforced concrete (RC) structures, in particular those strengthened with carbon-fibre-reinforced polymer (CFRP) sheets. The rationale is that the performance of such structural elements can be seriously affected by the de-bonding phenomenon. This is particularly relevant in the initial cracking stage, with the significant risk of a relevant reduction of their bearing capacity. In comparison with classical strain gauge sensors, the authors present numerically and experimental results obtained by using a Brillouin optical time-domain reflectometer (BOTDR) fibre sensor. The use of this type of sensor shows several advantages: although it might be less accurate than standard strain gauges, the BOTDR is not affected by the surface conditions, assuring, thus, steady measurements. Numerical finite elements simulations conducted with the commercial software LS-DYNA take into account all the aspects involved in the deflectional process, such as the mechanical properties of reinforced concrete and carbon fibres, the anisotropy of composite concrete, the orthotropic behaviour of polymeric sheets, the interface interactions, and the failure tiebreak contact model. Results coming from the four-point bending experiments conducted in displacement control over several specimens in different sheets bonding conditions highlight the prominent influence of de-bonding of CFRP in the initial cracking stage, while the ultimate failure state results uncorrelated. Moreover, experimental measurements and numerical analysis show a satisfactory match that grants the reliability of the latter as a predictive method. Thus, for all the aforementioned aspects, the methodology proposed by the authors constitutes a valid and robust technique for $\mathrm{RC}$ beams monitoring.

\section{Bayesian Calibration of Hysteretic Parameters with Consideration of the Model Discrepancy for Use in Seismic Structural Health Monitoring}

In the study reported in [8], the authors investigate model-driven seismic structural health monitoring procedures, based on a Bayesian uncertainty quantification framework. The variety of schemes and uncertainties that are typical of civil structures make the prediction of their actual mechanical behaviour and structural performance a difficult task. In this regard, computer simulations are useful engineering tools to design complex systems and assess their performance. These simulations aim at reproducing the underlying physical phenomena under investigation, providing a solution for the governing equations. However, accurate modelling of the structural systems requires the numerical models to be calibrated and validated with direct observations and measured experimental data. For this aim, the authors applied a Bayesian inference strategy to calibrate a nonlinear hysteretic Bouc-Wen model, derived from real data acquired on a monitored masonry building, in terms of both most probable values (MPV) and discrepancy posterior distribution. This pointed to the importance of correlating the choice of the discrepancy model function to the possible degradation amount and the characteristics of the external seismic input. The findings of the study define a non-arbitrarily of the choice of the discrepancy model. According to their findings, the selection of this model should be subordinated to the statistical nature of the external force (e.g., amplitude and frequency) and the statistical nature of the modal characteristics (e.g., natural frequencies) of a system, evaluated in operational conditions. For instance, for external forces with frequency content close to the natural frequencies of the system, there is a high chance of high degradation to occur, 
and thus a discrepancy model distribution close to a Gaussian distribution could bring trivial results. In conclusion, the authors make relevant considerations for the use of model-driven solutions for seismic structural health monitoring, especially for applications to masonry structures.

\section{Piezoelectric Electro-Mechanical Impedance (EMI) Based Structural Crack Monitoring}

In [9], the application of the piezoelectric electro-mechanical impedance (EMI) method is proposed as an effective active sensing approach to localise small cracks in beam- and plate-like structures. The integrity of the structure is investigated by analysing the imaginary peak frequency of the piezoelectric admittance spectrum. The rationale is that, in accordance with the coupled behaviour of the electro-mechanical systems, the occurrence of damage in the structure causes changes in both its piezoelectric and dynamic properties, with a close correlation between the modal resonance frequencies and the piezoelectric ones. This allows assuming the latter as a valid and sensitive indicator of the deviations from the pristine mechanical state. The proposed method is validated through numerical finite element (FE) and experimental simulations, where the crack size has fixed depth and width and variable length. The numerical analysis shows off the feasibility of the method and establishes it as a useful tool for the selection of the scanning bands in the piezoelectric admittance frequency spectrum due to the relationship with the harmonic response. The experimental results, conducted on aluminium specimens, are consistent with the numerical model and confirm the theoretical expectations of effectiveness in crack detection and growth monitoring. They also highlight a high accuracy level and stability when dealing with real-life, noisy observations. The decrement of local stiffness due to damage presence is related to lower admittance peak frequency, while the shift can be assumed as a feature for evaluating the severity of the crack and modulated through an increase in the detection frequency band. In conclusion, the proposed method has great potential as a compelling crack detection and monitoring strategy.

\section{Robust Structural Damage Detection Using Analysis of the CMSE Residual's Sensitivity to Damage}

A consistent improvement in damage identification is presented in [10]. The wellknown vibration-based method of cross-modal strain energy (CMSE) presents several intrinsic advantages in modal analysis with respect to traditional modal strain energy (MSE). For instance, it does not strictly require the same number of intact and damaged mode shapes. However, the resulting linear inverse problem is ill-conditioned, raising issues related to excessive perturbations propagation and incorporating some ineffective, otherwise counterproductive, redundant equations. To face this issue, the authors introduce a sensitivity analysis to identify and remove those inessential equations and enhance the effectiveness and the robustness of detection. Ancillary, two improvements of the iterative Tikhonov regularisation method have been proposed in the selection of the regularisation parameter of the adaptive strategy and in the formulation of the regularisation operator. These two aspected aimed at increasing both the rate of convergence and the accuracy. The effects of different damage levels and locations are investigated through noisy numerical simulations and confirmed by experimental validation. In all the investigated cases, the so-called robust cross-modal strain energy (RCMSE) method showed better performances than conventional CMSE. In particular, RMCSE outputs a considerably reduced number of false positives, which is an aspect of primary importance in the field of SHM. The robustness of results has been also confirmed by investigating the influence of different noise levels, with minor discrepancies at lower damage levels. Convincingly, the work presented in this paper progresses the effectiveness of MSE-based techniques. 


\section{A Novel Dense Full-Field Displacement Monitoring Method Based on Image Sequences and Optical Flow Algorithm}

An innovative methodology for deformation monitoring using image acquisition techniques is proposed in [11]. Specifically, a deep learning algorithm is applied, conjunctly to several vision technologies. This is intended to achieve a full-field displacement measurement, globally for the whole large-scale structure. This mainly overcome the typical issues of discrete target points observation, poor in the characterisation of the overall structure, while maintaining the advantages of long-range accuracy and cost-effectiveness. The proposed approach allows as well big data acquisition, which is essential for feeding machine learning algorithms. The designed noncontact remote sensing (NSR) device is able to acquire from multiple perspectives the time-space labelled static images sequences, according to the overlapped camera fields of view. Afterwards, edge detection, pixel virtual marker methods, and the scale-invariant feature transform (SIFT) algorithm are applied to holographically reconstruct the dense full-field displacement. The proposed device and method are applied to test a reduced-scale model of a self-anchored suspension bridge under several load and damage conditions. The bridge was equipped with dial gauges for displacement measurements and a numerical Finite Element model was developed for additional comparison. The experimental results show a level of accuracy high enough for engineering applications, reaching a maximum error of $12 \%$ with respect to the observation coming from conventional measurement devices and numerical predictions. Although this study represents only the first step towards a dense optical monitoring strategy and still requires further studies, the proposed methodology has great potentiality for real-time and long-term monitoring applications.

\section{Railway Wheel Flat Recognition and Precise Positioning Method Based on Multisensor Arrays}

The study published in [12] concerns the major problem of wheel defects detection and their long-term monitoring, to enhance a more sustainable maintenance planning of trains and rails while ensuring high standards of serviceability and safety. In particular, this work focuses on wheel flats, well-known defects responsible for anomalous impacts on the track that accelerate the degradation of both the track and the wheel itself. The proposed land-based measurement method consists of multisensor arrays, able to assess the condition of the wheels by evaluating the dynamic vertical strain response of the track during the train passage. A multibody dynamic system is numerically modelled using the finite element method; the rail web compression method is chosen to measure the wheel impact, due to its sensitivity to vertical strain, its stability to bending and torque moments caused by interfering lateral forces, and the low number of sensors required. The transverse, longitudinal and plane sensors layouts are also investigated to enhance accurate and unbiased measurements. Finally, the designed algorithm exploits multiple sensors data fusion to establish when the anomalous impacts occurred (by analysing the outliers space-time distribution), where those impacts occurred (through the sensors spatial correlation), and which wheel causes these abnormal fluctuations (by associating average speed with time and position to individuate the impact processes). The subsequent offline experimental validation confirmed the capability of the algorithm to effectively recognise and locate the presence of wheel flats. The main contribution of this paper is the proposal of a real-time monitoring solution that, for its effectiveness and its feasibility, is able to easily detect wheel flat; this can concretely improve the maintenance operations for railroad owners and operators.

\section{Monitoring and Analysis of Dynamic Characteristics of Super High-Rise Buildings Using GB-RAR: A Case Study of the WGC under Construction, China}

The importance of monitoring the dynamic characteristics of skyscrapers and tall buildings is well outlined in [13], as extreme displacements can cause severe damage to the structures or compromise their operational safety, especially during the construction phase. This research describes a field application of displacement measurements with the 
technique of interferometric ground-based real aperture radar (GB-RAR), able to overcome the limitations of classical methodologies, such as the need to place sensors, resorting to expensive devices. It also compares favourably, from a cost-efficient point of view, with satellite-based imaging technologies, which are affected by low resolution, potential misrepresentation due to atmospheric effects, and delay in delivery of the results. The main application of this study consists of monitoring a high-rise building under construction in mainland China. The proposed strategy reached a sub-millimetric level of accuracy in measurements. In the paper, the influence of temperature is investigated through a meteorological station. The procedure adopted by the authors include also several correction methods to correct the intrinsic and extrinsic influence of the acquired signal data. In particular, a windowing procedure is used to eliminate sidelobe effects. Gross error due to external vibrations and environmental factors are detected and removed and a wavelet denoising procedure is applied to the observations. Although working in a construction site context, characterised by the presence of several interfering structures (such as service platforms, cranes, etc...) and disturbing environmental elements like wind, sunlight and temperature, the methodology proposed by the authors is effectively capable to identify trajectories and displacements of the buildings, confirming to be a highly accurate, low-cost and non-invasive valid solution, even in presence of construction vibrations and unfavourable conditions.

\section{State-of-the-Art Review on Determining Prestress Losses in Prestressed Concrete Girders}

The monitoring of prestress losses in prestressed concrete (PC) girders is fundamental to preserve the structural integrity of bridges. Indeed, the deleterious effects of prestress losses require the elapse of a long time to emerge and, thus, their identification and predictive maintenance result to be very challenging. A state-of-art review on this topic is presented in [14], with a particular focus on the existing non-destructive testing methods and related strategies. The authors firstly review the context of the application for PC elements. Here, they propose an overview of previous works aimed to measure the prestressing force and to predict related losses, considering over 30 articles concerning different experimental and numerical methodologies. The following sections extensively collect more than 60 papers of experimental and numerical research works, ranging over different metrics (i.e., mechanical parameters, vibrational features, etc.) and approaches (considering destructive, semi-destructive, and non-destructive testing options). Finally, the study focuses on static NDT methods, remarking the higher reliability and sensitiveness to prestressing losses in comparison with dynamic techniques. The overview offered for such an important topic will be essential to both academic researchers and practitioners.

\section{Application of the Subspace-Based Methods in Health Monitoring of Civil Structures: A Systematic Review and Meta-Analysis}

Subspace system identification (SSI) methods have been widely studied and applied in the last two decades, investigating both mechanical and civil structures with numerical and experimental analyses. Since its large spread and abundance of academic research papers, the need for a systematic literature review is faced in [15]. The authors follow the Preferred Reporting Items for Systematic Reviews and Meta-Analyses (PRISMA) approach to conduct rigorous selection, screening, classification and examination of reviewed works, summarising at the end of the process a total of 69 articles from 31 international journals published in the period 2008-2019. Criteria of classification include the typology of test structures, nature of processing algorithms, a-priori knowledge of the input and/or the output, and influence of operational and environmental conditions. Moreover, the authors propose several comparisons and considerations on different methods, discussing the advantages and disadvantages of the different techniques. Conclusively, the extensive literature review presented by the authors poses the basis for further studies in the field of subspace-based methods, comprehensively outlining research gaps and future perspectives to enhance future developments. 
Funding: This research received no external funding.

Acknowledgments: This Special Issues collected the efforts of all the authors, reviewers, and members of the Editorial Office of Applied Sciences. We would like to thank all the professional contributions to this publication. The Special Issue benefited from the coordination efforts and the support from Marco Civera and Davide Martucci.

Conflicts of Interest: The author declares no conflict of interest.

\section{References}

1. Martucci, D.; Civera, M.; Surace, C. The Extreme Function Theory for Damage Detection: An Application to Civil and Aerospace Structures. Appl. Sci. 2021, 11, 1716. [CrossRef]

2. Roy, R.; Gherlone, M.; Surace, C.; Tessler, A. Full-Field Strain Reconstruction Using Uniaxial Strain Measurements: Application to Damage Detection. Appl. Sci. 2021, 11, 1681. [CrossRef]

3. Bombarda, D.; Vitetta, G.M.; Ferrante, G. Rail Diagnostics Based on Ultrasonic Guided Waves: An Overview. Appl. Sci. 2021, 11, 1071. [CrossRef]

4. $\quad$ Kwon, D.-S.; Jin, C.; Kim, M.; Koo, W. Mooring-Failure Monitoring of Submerged Floating Tunnel Using Deep Neural Network. Appl. Sci. 2020, 10, 6591. [CrossRef]

5. Żurawski, M.; Zalewski, R. Damping of Beam Vibrations Using Tuned Particles Impact Damper. Appl. Sci. 2020, 10, 6334. [CrossRef]

6. Chmelko, V.; Garan, M.; Šulko, M.; Gašparík, M. Health and Structural Integrity of Monitoring Systems: The Case Study of Pressurized Pipelines. Appl. Sci. 2020, 10, 6023. [CrossRef]

7. Hong, K.-N.; Shim, W.-B.; Yeon, Y.-M.; Jeong, K.-S. Strain Response Characteristics of RC Beams Strengthened with CFRP Sheet Using BOTDR. Appl. Sci. 2020, 10, 6005. [CrossRef]

8. Ceravolo, R.; Faraci, A.; Miraglia, G. Bayesian Calibration of Hysteretic Parameters with Consideration of the Model Discrepancy for Use in Seismic Structural Health Monitoring. Appl. Sci. 2020, 10, 5813. [CrossRef]

9. Wang, T.; Tan, B.; Lu, M.; Zhang, Z.; Lu, G. Piezoelectric Electro-Mechanical Impedance (EMI) Based Structural Crack Monitoring. Appl. Sci. 2020, 10, 4648. [CrossRef]

10. Xu, M.; Wang, S.; Guo, J.; Li, Y. Robust Structural Damage Detection Using Analysis of the CMSE Residual's Sensitivity to Damage. Appl. Sci. 2020, 10, 2826. [CrossRef]

11. Deng, G.; Zhou, Z.; Shao, S.; Chu, X.; Jian, C. A Novel Dense Full-Field Displacement Monitoring Method Based on Image Sequences and Optical Flow Algorithm. Appl. Sci. 2020, 10, 2118. [CrossRef]

12. Zhou, C.; Gao, L.; Xiao, H.; Hou, B. Railway Wheel Flat Recognition and Precise Positioning Method Based on Multisensor Arrays. Appl. Sci. 2020, 10, 1297. [CrossRef]

13. Zhou, L.; Guo, J.; Wen, X.; Ma, J.; Yang, F.; Wang, C.; Zhang, D. Monitoring and Analysis of Dynamic Characteristics of Super High-rise Buildings using GB-RAR: A Case Study of the WGC under Construction, China. Appl. Sci. 2020, 10, 808. [CrossRef]

14. Bonopera, M.; Chang, K.-C.; Lee, Z.-K. State-of-the-Art Review on Determining Prestress Losses in Prestressed Concrete Girders. Appl. Sci. 2020, 10, 7257. [CrossRef]

15. Shokravi, H.; Shokravi, H.; Bakhary, N.; Heidarrezaei, M.; Rahimian Koloor, S.S.; Petrů, M. Application of the Subspace-Based Methods in Health Monitoring of Civil Structures: A Systematic Review and Meta-Analysis. Appl. Sci. 2020, 10, 3607. [CrossRef] 